\title{
Electric-field modulation of liquid crystal structures in contact with structured surfactant monolayers
}

\author{
Pau Guillamat, Francesc Sagués, and Jordi Ignés-Mullol \\ Institut de Nanociència i Nanotecnologia $\left(I N^{2} U B\right)$ and Departament de Química Física, \\ Universitat de Barcelona, Martí i Franquès 1, 08028 Barcelona, Catalonia, Spain
}

(Received 20 December 2013; published 27 May 2014)

\begin{abstract}
We present experiments in which we use an electric field to switch between different configurations in the cellular patterns induced in a confined nematic liquid crystal by the contact with a surfactant monolayer that features lateral order and surface defects. By using different combinations of far-field alignment and mesogen dielectric anisotropy, we unravel the nature and stability of point defects and disclinations resulting from the hybrid boundary conditions.
\end{abstract}

DOI: 10.1103/PhysRevE.89.052510

PACS number(s): 61.30.Jf, 61.30.Hn, 61.30.Pq, 61.30.Gd

\section{INTRODUCTION}

Topological defects are inherent in liquid crystals (LCs) [1-3], and they typically arise in confined geometries after a quench of an electric or a magnetic field, due to incompatible boundary conditions at the outer surfaces [4-6], or as a consequence of the inclusion of colloidal objects in the LC matrix [7-12]. The ability to control these defects offers opportunities for alternative devices and materials [13-17]. The proliferation of disclination lines can be controlled by patterning the bounding surfaces of LC cells. For instance, this has been achieved through mechanically $[18,19]$ or optically [20-23] scribing polymeric or other thin films [24]. On the other hand, LCs in contact with structured self-assembled surfactant monolayers have been demonstrated to propagate the order present in the two-dimensional soft films well into the bulk LC [25,26]. In particular, the transfer of monolayers previously assembled at the air/water interface onto a glass plate has been demonstrated to be a good strategy for LC alignment [27], even with mesogens for which traditional alignment techniques fail [28]. The contact with surfaces patterned by self-assembled structures featuring a well-defined and robust tilt-orientational order results in a large-scale self-organization of the LC director that would be difficult to achieve by the artificial methods related above.

In a previous work [29], we built hybrid nematic liquid crystal cells in which one of the plates was coated with a Langmuir-Blodgett (LB) film [30] that was previously self-assembled by spreading an insoluble surfactant at the air/water interface. We prepared this precursor Langmuir monolayer under conditions that lead to the formation of circular submillimeter isolated domains rich in the elongated form of our surfactant (trans isomer), which spontaneously phase-separates from the bent cis form (Fig. 1). Surfactant molecules inside the circular domains self-assemble into a two-dimensional mesophase [31], with molecules uniformly tilted with respect to the interface normal and featuring a spatial distribution of the in-plane azimuth. Surrounding the circular domains, the cis-rich matrix is disordered. The mesophase domains feature surface defects that propagate into the LC bulk. The imposed uniform far-field alignment leads to a universal defect healing scenario in the form of arch-shaped disclinations that propagate into the bulk LC, either in the form of a single twist-escaped or double singular disclination.
Transformation between the two configurations was only possible when mediated by a transition into the isotropic and back into the nematic phase, which led to the irreversible conversion of single-arch into double-arch disclinations, and we argued that the reverse transformation was prevented by a kinetic barrier.

In this work, we use electric fields to probe the stability of the structures arising in the different defect healing scenarios when the same patterned surfactant monolayers with lateral order and point defects are used as aligning layers of nematic LC's. We show that twist-escaped disclinations are metastable against singular bulk disclinations, whose structure is different from the double-arch disclinations obtained after cycling through the isotropic phase. We demonstrate that the latter structures are surface disclinations that remain unperturbed by the application of external fields. In contrast, twist-escaped and singular bulk disclinations can be transformed into each other by a suitable choice of mesogen, far-field alignment, and applied field.

\section{EXPERIMENTAL SECTION}

\section{A. Substrate preparation}

Indium tin oxide (ITO) -coated glass slides or coverslips (100 $\Omega$ /square, VisionTek Systems, Ltd.) are cut to the desired size and degreased with a diluted soap solution (Micro-90 concentrated cleaning solution from Sigma-Aldrich in Milli-Q water, $1 \% \mathrm{w} / \mathrm{w}$ ), Milli-Q water (both with 2 min sonication), acetone (lab grade), ethanol (96\% v/v p.a., Panreac), and Milli-Q water again (the three with $10 \mathrm{~min}$ sonication). The clean plate is dried in a stream of nitrogen and baked at $130{ }^{\circ} \mathrm{C}$ for $10 \mathrm{~min}$ to eliminate traces of water. To render the ITO surface hydrophilic for Langmuir-Blodgett monolayer deposition, the clean substrate is pretreated in a mixture of 5:1:1 (v/v/v) purified $\mathrm{H}_{2} \mathrm{O} / \mathrm{H}_{2} \mathrm{O}_{2}(33 \%$ w/v, Panreac $) / \mathrm{NH}_{4} \mathrm{OH}$ (28-30\%, ACS reagent, Sigma-Aldrich) at $70-80^{\circ} \mathrm{C}$ for $1.5 \mathrm{~h}$ to maximize the presence of hydroxyl groups on the surface. The substrate is then washed with a copious amount of Milli-Q water and dried with nitrogen. After $20 \mathrm{~min}$ baking at $50^{\circ} \mathrm{C}$, the hydroxylated ITO is immersed in a solution containing $1 \%$ (v/v) APTES [(3-Aminopropyl)triethoxysilane] (purity $\geqslant$ $98 \%$, Sigma-Aldrich) in toluene (reagent grade, Scharlau) for $1 \mathrm{~h}$ at $70-80^{\circ} \mathrm{C}$ to form a silane monolayer. Finally, the plate is 


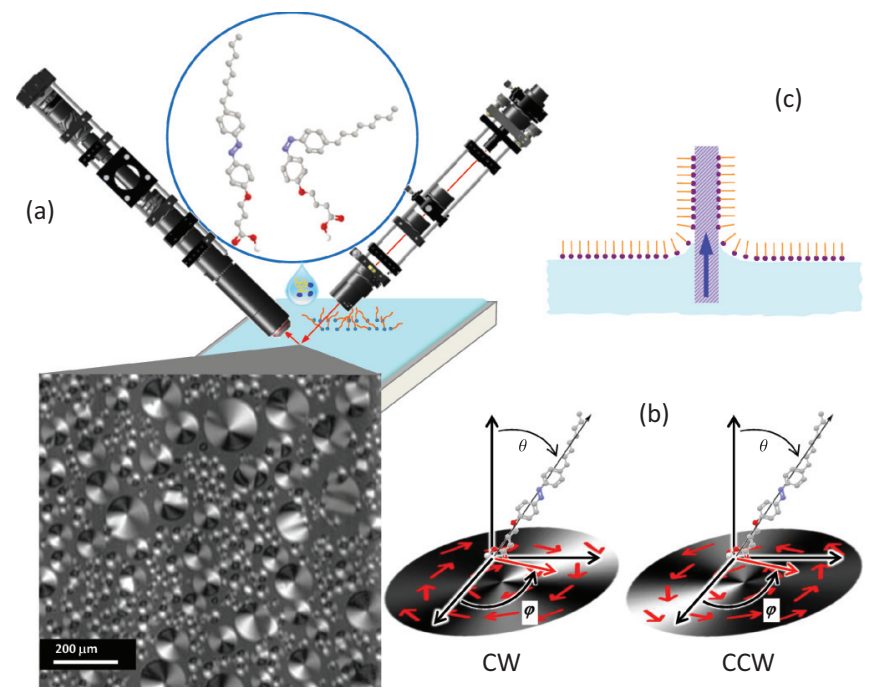

FIG. 1. (Color online) (a) Patterned Langmuir monolayers are prepared by the spontaneous phase separation of the cis and trans isomers of an azobenzene surfactant spread at the air/water interface. Brewster angle microscopy reveals the formation of mesophase domains where hydrophobic chains have a uniform tilt angle $(\theta)$ and a spatially varying azimuth $(\varphi)$, which defines two mirror image configurations of the in-plane molecular order (b). The patterned film is transferred to a solid plate by means of the Langmuir-Blodgett (LB) technique (c).

rinsed twice with toluene and cured under a vacuum at room temperature for $12 \mathrm{~h}$. This protocol results in an ITO surface grafted with terminal $\mathrm{NH}_{2}$ groups, which makes the surface hydrophilic, acquiring a positive charge in aqueous solution; this is the optimal situation for the subsequent transfer of a carboxylic-acid surfactant monolayer. In the case of plain glass, the substrate can be cleaned and activated (hydroxylated) at the same time using piranha solution, $1: 3 \mathrm{H}_{2} \mathrm{O}_{2}(33 \% \mathrm{w} / \mathrm{v}$, from Panreac)/ $\mathrm{H}_{2} \mathrm{SO}_{4}$ (lab grade) v/v, rinsed with Milli-Q water, and baked as described above to remove traces of water.

For hybrid nematic liquid crystal (NLC) cells, we prepare complementary plates with either homogeneous planar or homeotropic anchoring. For planar anchoring, the plate is cleaned as described above and subsequently spin-coated with polyimide (PI-2555, HD-Microsystems) using a syringe with a $0.22 \mu \mathrm{m}$ filter to spread the polyimide solution followed by spinning at $600 \mathrm{rpm}$ for $3 \mathrm{~s}$ and at $2000 \mathrm{rpm}$ for $60 \mathrm{~s}$ (Chemat $\mathrm{KW}-4 \mathrm{~A}$ ), baking at $300{ }^{\circ} \mathrm{C}$ for $2 \mathrm{~h}$ on a hot plate, and then rubbing unidirectionally with a velvet cloth. For homeotropic anchoring, the clean surface is functionalized with Dimethyloctadecyl[3-(trimethoxysilyl)propyl]ammonium chloride (DMOAP, Sigma-Aldrich) by immersing it in a very diluted DMOAP (Sigma Aldrich) aqueous solution (0.01 M) for $30 \mathrm{~min}$ at room temperature. The plate is then rinsed with Milli-Q water, dried with a stream of nitrogen, and heated at $130^{\circ} \mathrm{C}$ for $1.5 \mathrm{~h}$ to favor the formation of a self-assembled monolayer.

\section{B. Langmuir-Blodgett film preparation}

LB films are prepared by means of the transfer of Langmuir monolayers on a substrate (Fig. 1). Spreading solutions of the custom-synthesized photosensitive surfactant 4-[4[4-octylphenyl/azo]-phenoxy]butanoic acid (8Az3COOH) are prepared by dissolving the solute in chloroform (stabilized with $0.75 \%$ ethanol, J.T. Baker) at a surfactant concentration of $0.5 \mathrm{mg} \mathrm{mL}^{-1}$. Prior to monolayer preparation, the solution is irradiated with a uv lamp (Vilber Lourmat VL-6.L, $\lambda=365$ $\mathrm{nm}$ ) for $10 \mathrm{~min}$ to promote the photoisomerization to the metastable isotropic cis form. A hydrophilic plate is immersed in the water subphase of a Langmuir trough (minitrough, KSV NIMA), thermostatted at $25^{\circ} \mathrm{C}$, and then the solution is spread dropwise using a microsyringe, until reaching a surface pressure of $1 \mathrm{mN} \mathrm{m}^{-1}$. Relaxation at a constant area under room light for 60 min leads to a spontaneous isomerization into the trans form and to the formation of structured circular submillimeter trans-rich domains surrounded by the isotropic cis-rich matrix. A custom-made Brewster angle microscope is used to monitor the size and inner texture of the domains. The substrate is subsequently lifted up at $8 \mathrm{~mm} \mathrm{~min}^{-1}$ by means of the minitrough dip coater, resulting in a monolayer transfer that preserves the lateral organization present at the air/water interface.

\section{Liquid crystal cell preparation}

NLC cells are prepared by assembling two parallel plates $(1.5 \times 2.5 \mathrm{~cm})$ with polyethylene terephthalate $(\mathrm{PET})$ sheets (23 $\mu \mathrm{m}$, Mylar, GoodFellow) or monodisperse silica microrods (5.0 $\mu \mathrm{m}$ diameter, Nippon Electric Glass Co.) between them, acting as spacers. The parallel plates are glued using epoxy glue (Hysol, Henkel), which confers robustness to the system. ITO-coated plates are used when an electric-field normal to the plates is desired. For an in-plane electric field, a pair of copper wires ( $25 \mu \mathrm{m}$ in diameter) running parallel to the filling direction are embedded inside the cell with a separation of $1 \mathrm{~mm}$ between them, serving also as spacers. The cells are filled by capillarity with NLCs, either E7 (Merck) for positive dielectric anisotropy $\left(\varepsilon_{a}>0\right)$ or MLC-7029 (Merck) for $\left(\varepsilon_{a}<\right.$ $0)$. Unless otherwise stated, filling direction is kept parallel to the rubbing in cells having substrates with planar anchoring. To perform fluorescent confocal polarizing microscopy (FCPM) observations, the anisotropic dye N-NBis(2,5-ditert-butyl-phenyl)-3,3,9,10-perylenedicarboximide (BTBP) is mixed with the LC at low concentrations (0.01 wt. \%).

\section{Microscopy techniques and electric field modulation}

Polarized light microscopy is performed with a Nikon Eclipse 50iPol upright microscope. Images are acquired with an AVT Marlin 131B CMOS digital video camera, and further processing is carried out with the software IMAGEJ [32]. Unless otherwise noted, micrographs are obtained with the samples between crossed polarizers, as illustrated in Fig. 2. FCPM imaging has been performed with an Olympus Fluoview FV300 system (Scientific and Technological Centers, Universitat de Barcelona). The electric field has been applied with a function generator (Agilent Technologies DSO-X 2002A) connected to a power amplifier (TREK PZD700) and monitored with an oscilloscope (integrated in the current generator). We apply square wave ac fields ( $50 \%$ duty cycle) with $f=1.0 \mathrm{kHz}$ and amplitudes in the range $0-10 \mathrm{~V}$ in cells 

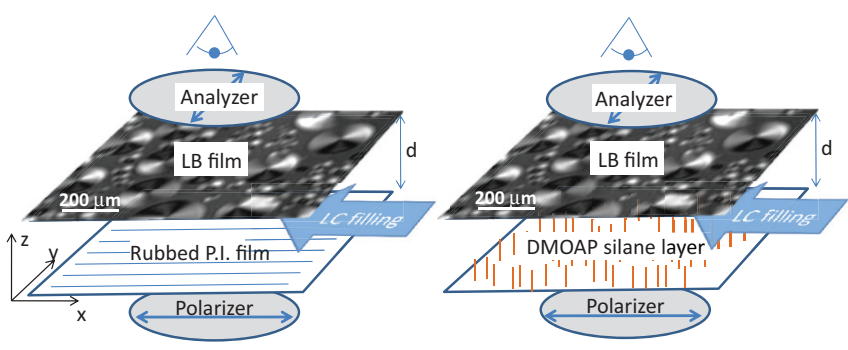

FIG. 2. (Color online) Hybrid nematic LC cells assembled by combining a LB-coated plate and a complementary plate that induces homogeneous anchoring, either homeotropic or planar. Cells are observed between crossed polarizers, with the LB-coated plate above. If planar anchoring is used, the rubbing direction is parallel to the polarizer and to the flow-filling direction.

with hydrophylized ITO plates or 0-500 V for an in-plane field between embedded wires.

\section{RESULTS AND DISCUSSION}

\section{A. Formation of the twist-escaped disclination and persistence of the boojum defects}

Flow filling in the nematic phase with planar anchoring on the complementary plate results in the formation of two boojums (surface defects) inside a circular LB domain and an arch-shaped twist-escaped disclination subtended between them [29] [see Figs. 3(a)-3(c)]. One of the boojums has strength $s=+1$ and is located at the center of the domain. It is a direct consequence of the coupling between the three-dimensional nematic director field and the in-plane order of the surfactant monolayer. The other boojum has strength $s=-1$ and is located on the domain periphery, at a position radially perpendicular to the local flow filling direction. The degeneracy between the two possible radial directions is broken by the underlying asymmetry of the surfactant monolayer domain. The director field is twisted across the cell gap by a different amount depending on the relative orientation between the local tilt of the surfactant molecules and the cell filling direction. In the region where they are antiparallel, twist distortion is maximum and changes sign across the twist-escaped disclination that develops [Fig. 3(c)]. The presence of the two oppositely charged surface defects is a topological requirement in order for the nematic director field to heal the singularity present at the center of the monolayer domain as it evolves toward the homogeneous complementary plate. Upon transition to the isotropic and back into the nematic phase, this configuration is irreversibly transformed into two singular surface disclinations [Figs. 3(e) and 3(f)] that are unaltered by the application of an electric field.

Flow during cell filling results in planar alignment of $\mathbf{n}(\mathbf{r})$ away from the LB-coated surface, regardless of the boundary conditions at the complementary plate. As a result, the combination of boojums and twist-escaped disclination described above is a universal scenario of defect healing between the two-dimensional ordered surfactant domains and the homogeneous far-field anchoring. The resulting configuration, however, may be unstable if boundary conditions on the complementary plate are different from planar or do not
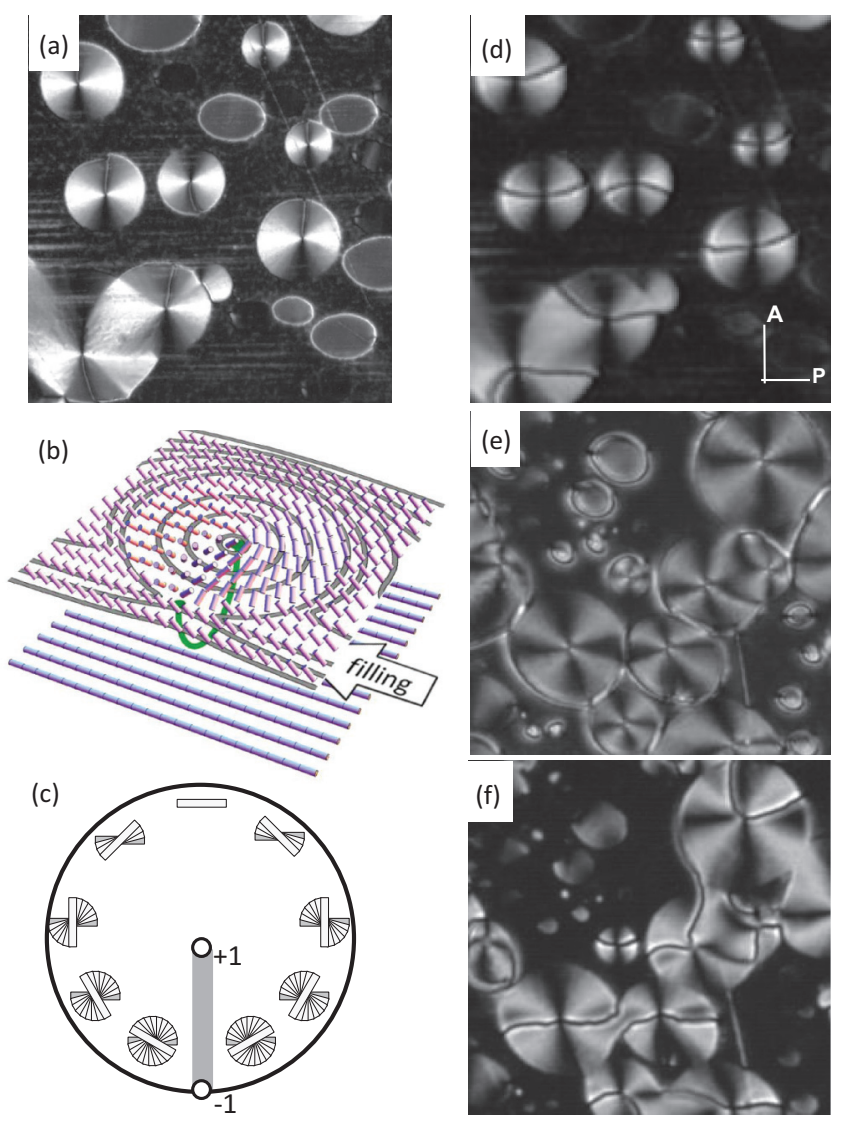

FIG. 3. (Color online) (a) Polarizing optical micrograph of a hybrid cell with planar far-field anchoring filled with the NLC E7 (cell gap $5 \mu \mathrm{m}$ ). The LB film has been transferred on plain glass. (b) Healing of the point defect on a domain with counterclockwise surfactant tilt orientation results in the formation of a south-facing arch-shaped twist-escaped disclination (green line) pinned on two boojum defects. (c) Top view of the domain in (b) with the two integer boojum defects and a $2 \mathrm{D}$ projection representing the reorientation of the director field across the cell gap, showing a nonhomogeneous twist distortion. The pattern changes irreversibly after transition into the isotropic and back into the nematic phase (d). For homeotropic farfield, the configuration can be free from the twist-escaped disclination inside the domain (e), but it develops singular disclinations after cycling into the isotropic phase (f). Panels containing micrographs are $500 \mu \mathrm{m}$ wide.

coincide with the flow-filling direction. For instance, in thin cells (less than $10 \mu \mathrm{m}$ ) with homeotropic anchoring on the complementary plate, the steady-state configuration has no disclination inside the domain [Fig. 3(e)] [29]. The relaxation from flow-aligned planar to homeotropic in nematic cells is known to proceed trough the propagation of $s=-1 / 2$ wedge disclinations [33]. When such a disclination overlaps with a domain featuring a twist-escaped disclination (Fig. 4), the wedge disclination binds to the $s=-1$ boojum, which detaches from the surface (i.e., it becomes a hedgehog). The resulting $s=-1$ bulk defect travels toward the central $s=+1$ defect as the twist-escaped disclination unfolds. The bulk edge disclination finally traverses the domain, and the Maltese cross texture expected for hybrid anchoring between circular planar and homeotropic is observed, although a memory of the 

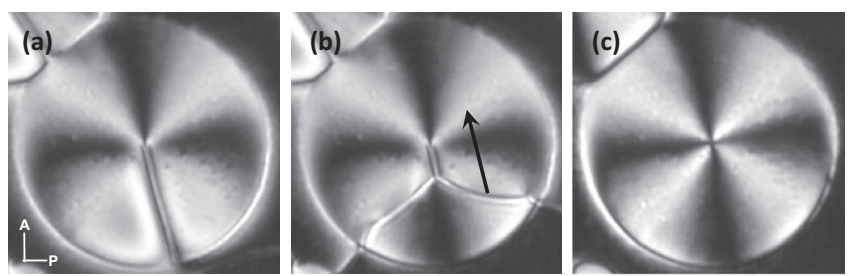

FIG. 4. Hybrid cell $(\mathrm{gap}=5 \mu \mathrm{m})$ with the LB film transferred on plain glass and homeotropic far-field alignment. (a) Upon filling with E7, cells develop a twisted escaped disclination due to flow alignment. (b) For thin cells, this structure is unstable and disappears when an $s=-1 / 2$ wedge disclination separating homeotropic and planar regions moves over the domain, leaving a disclination-free four-brush configuration behind (c). The width of each frame is $100 \mu \mathrm{m}$. See Video4 in the supplemental material [34].

boundary boojum seems to be preserved, as suggested by the asymmetric shape of the Maltese cross.

This flow-imprinted memory of surface defects can be tested using an electric field to impose a planar far-field alignment in a direction different from the flow-filling one. In Fig. 5, a hybrid cell with homeotropic boundary conditions on the complementary plate is filled with the liquid crystal E7 $\left(\varepsilon_{a}>0\right)$. An in-plane field is applied by means of a pair of copper wires running along the horizontal direction in the micrographs. Although the cell is filled in the direction parallel to the wires, twist-escaped disclinations are typically oriented at $45^{\circ}$ instead of the expected $90^{\circ}$, presumably because flow filling was distorted by capillary effects due to the presence of the wires [Fig. 5(a)]. An ac electric field $(f=1 \mathrm{kHz}$, $\left.0.2 \mathrm{~V} \mu \mathrm{m}^{-1}\right)$ applied between the wires aligns $\mathbf{n}(\mathbf{r})$ in-plane, north-to-south in Fig. 5(b). Inside the circular domain, the twist-escaped disclination has rotated to be perpendicular to the planar far-field direction. As it approaches the domain boundary, the disclination bends and wraps along the boundary until it meets the position of the original $s=-1$ boojum. Extinction of the electric field can result in the disappearance of the twist-escaped disclination [such as in the example in Fig. 5(c)], although the boundary boojum is clearly discernible. Subsequent ON-OFF cycling of the electric field results in a transition between the two stable configurations in Figs. 5(b)
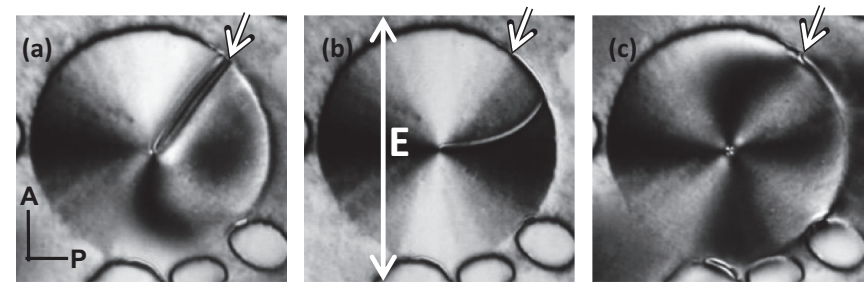

FIG. 5. Hybrid cell $(\mathrm{gap}=25 \mu \mathrm{m})$ with the LB film transferred on plain glass, homeotropic far-field alignment. (a) Flow filling with E7 (right-to-left in the image) leads to the formation of persistent twistescaped disclination pinned on two boojum defects. (b) Application of an in-plane E-field as illustrated leads to LC alignment top-tobottom, and to the reorientation of the twist-escaped disclination. (c) Extinction of the electric field may lead to the disappearance of the disclination. The position of the persistent $s=-1$ boojum is indicated by an arrow in all panels. The width of each frame is $100 \mu \mathrm{m}$. See Video5 in the supplemental material [34]. and 5(c). Notice that, unlike the formation of the disclination due to flow-alignment, there will now be two equivalent radial directions where the disclination is perpendicular to the far-field. In situations like the one illustrated in Fig. 5, the asymmetry in the position of the boundary boojum makes the eastward radial direction more favorable. In cases in which the surface boojum points precisely to the north or to the south, subsequent ON-OFF cycling of the electric field results in random positioning (eastward or westward) of the twist-escaped disclination.

These observations help to understand that hybrid planar cells, always filled parallel to the buffing direction, may exhibit twist-escaped disclinations that have curved shapes. Since cell filling is not controlled with precision, the local direction of the nematic flow velocity may differ significantly from the steady-state planar far-field direction. As a consequence, the $s=-1$ boojum is created along a radial direction that is offset from the one dictated by the buffing direction, resulting in the formation of disclinations with curved in-plane projections. The relevance of the persistent $s=-1$ boojum can be further put into evidence in a hybrid cell with homeotropic anchoring on the complementary plate and filled with a NLC with $\varepsilon_{a}<$ 0 (data not shown). Application of an electric field across the cell results in a degenerate planar far-field. The formed twist-escaped disclinations are now observed to be straight, but pointing to a direction that may differ significantly in different regions of the sample, thus effectively mapping the unevenness of the cell filling.

The experiments above illustrate the persistence of the imprint left on the anchoring surface by flow alignment effects. This memory is only erased when the mesogen is transited to the isotropic phase and back into the nematic [Fig. 3(d)]. In the present case, the precise placement of the boundary $s=-1$ boojum is sufficient to determine the configuration of the twistescaped pattern, thus providing multiple scenarios to transit between two complex $\mathbf{n}(\mathbf{r})$ patterns by means of an electric field.

\section{B. Instability of the twist-escaped disclination}

In our earlier publication [29], we compared the stability of the single twist-escaped disclination versus the double singular disclinations obtained after cycling the sample into the isotropic phase. Our estimations concluded that the free energy of the two configurations has the same order of magnitude, and we argued that the fact that they cannot be converted into each other (even after manipulation with optical tweezers) was likely due to a high energy barrier between the two states. Indeed, samples prepared in the nematic phase can maintain the single-arch configurations for extended periods of time (days, even weeks). Eventually, however, the structure of $\mathbf{n}(\mathbf{r})$ evolves so that increasingly large regions lose the twistescaped disclinations in favor of a proliferation of singular disclinations that cross domains in different directions, on average parallel to the planar far-field (Fig. 6). Such a process remained largely unexplored in our previous report, as it was ascribed to unavoidable sample degradation.

Although these singular disclinations are reminiscent of those obtained after cycling into the isotropic phase, the latter are surface disclinations unperturbed by the application of electric fields, while the former are bulk disclinations that can 


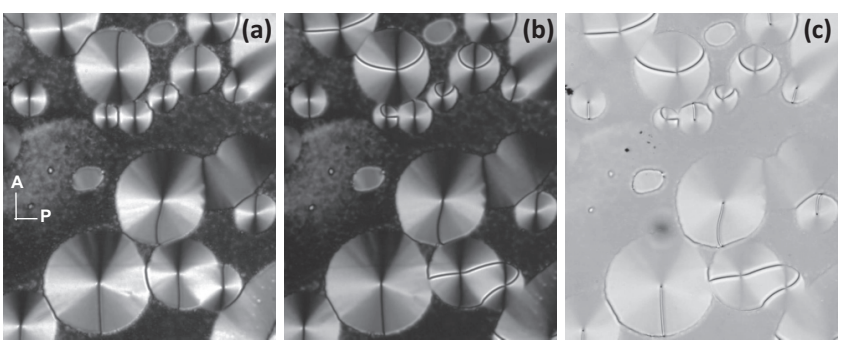

FIG. 6. Spontaneous relaxation of twist-escaped disclinations (a) into singular bulk disclinations (b) after an elapsed time of $18 \mathrm{~h}$ in a cell filled with E7. In (c), the same image is shown without a polarizer, evidencing the different nature of escaped and singular disclinations, and the presence of boojums. The LB has been transferred to plain glass, with planar far-field conditions and a cell gap of $23 \mu \mathrm{m}$. The width of each frame is $250 \mu \mathrm{m}$.

be created and destroyed by means of an electric field. In Fig. 7, a hybrid sample with planar alignment in the complementary plate is switched to homeotropic far field by means of an electric field. We show the final homeotropic texture for a domain with the twist-escaped disclination, and a domain where the latter had spontaneously relaxed into singular bulk disclinations. The two textures are indistinguishable and have no disclination inside the domain. On the other hand, for domains with the double-arch surface disclinations [Fig. 7(c)],
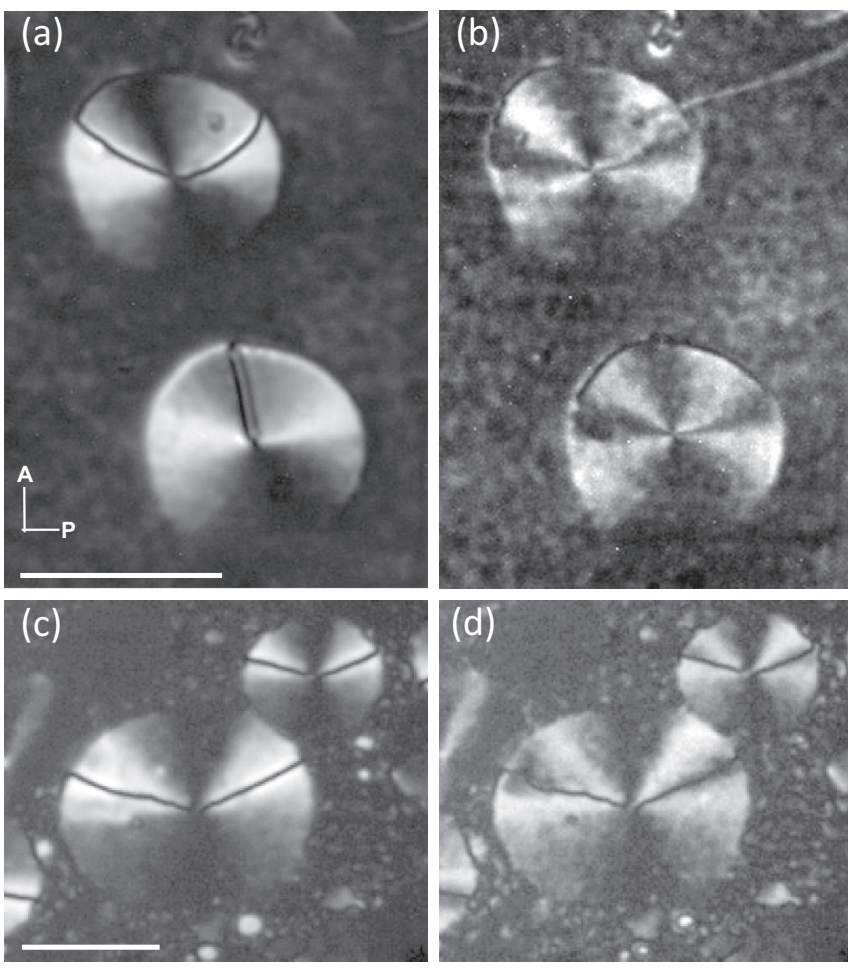

FIG. 7. Optical micrographs of a hybrid cell with homogeneous planar far field before $(a, c)$ and after $(b, d)$ the application of a $6 \mathrm{~V}$ ac electric field perpendicular to the cell. The sample in (c) has been previously transited into the isotropic and back into the nematic phase. The LB film has been transferred on APTES-functionalized ITO glass. The cell is filled with E7 and the gap is $5 \mu \mathrm{m}$. The line segments are $100 \mu \mathrm{m}$ long.
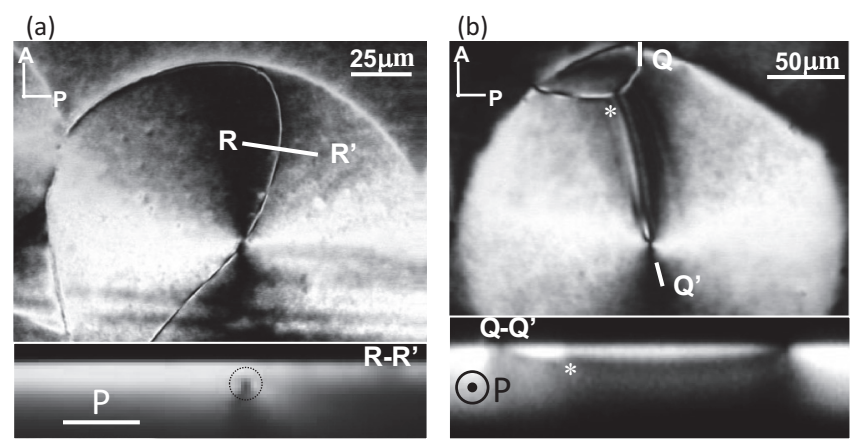

FIG. 8. Optical micrographs (top) and FCPM cross sections (bottom) across a singular bulk disclination line (a) and along a twist-escaped disclination that is evolving into a bulk disclination (b). The star symbol marks the place where the two types of disclinations meet. The core of the disclination in (a) is encircled by a circle in the cross section. The cell is filled with E7, and its gap is $23 \mu \mathrm{m}$.

the transition to homeotropic alignment leaves the surface disclinations unperturbed [Fig. 7(d)].

In our previous report [29], we used FCPM imaging to reveal that these surface disclinations are pinned on boojum defects and are attached to the surfactant-coated surface. For comparison, we have performed here FCPM measurements on samples obtained after the spontaneous relaxation of twist-escaped disclinations (Fig. 8), and we show that the resulting singular disclinations are indeed embedded in the nematic bulk. Occasionally, this relaxation process is perturbed by pinning on some irregularity, leaving the twist-escaped disclination linking the central boojum defect with a disclination loop. FCPM imaging shows that, in this configuration, the twist-escaped disclination is anchored between the central boojum and a point defect clearly separated from the surface at the receding end, where the twist-escaped disclination meets the disclination loop (Fig. 8).

Application of suitable electric field cycling allows us to switch between the single twist-escaped and the double bulk singular disclination configurations, and to observe the pathway of spontaneous relaxation of the metastable single disclination configuration. In Fig. 9, we present a region in a sample with planar far-field anchoring where the twist-escaped disclinations have spontaneously relaxed into singular bulk disclinations. Application of a strong vertical electric field results in the formation of the common homeotropic texture, as explained above (see Fig. 7). Slowly ramping down the electric field results in the recovery of the original texture that features singular bulk disclination only. A sudden quench, however, results in the formation of an unstable twist-escaped disclination [Fig. 9(d)], which unfolds into two singular disclinations from the outer part of the domain inward toward the $s=+1$ boojum, on which the twist-escaped disclination is pinned at all times [Figs. 9(d)-9(f)]. Once the process is completed, the configuration in Fig. 9(a) is recovered, with domains featuring singular disclinations than run along a diameter roughly perpendicular to the original escaped disclinations, and remain pinned to the central boojum defects.

We can understand the unfolding of the twist-escaped disclination by noticing that it leads to a relaxation of the twist distortion imposed across the cell gap by the hybrid 

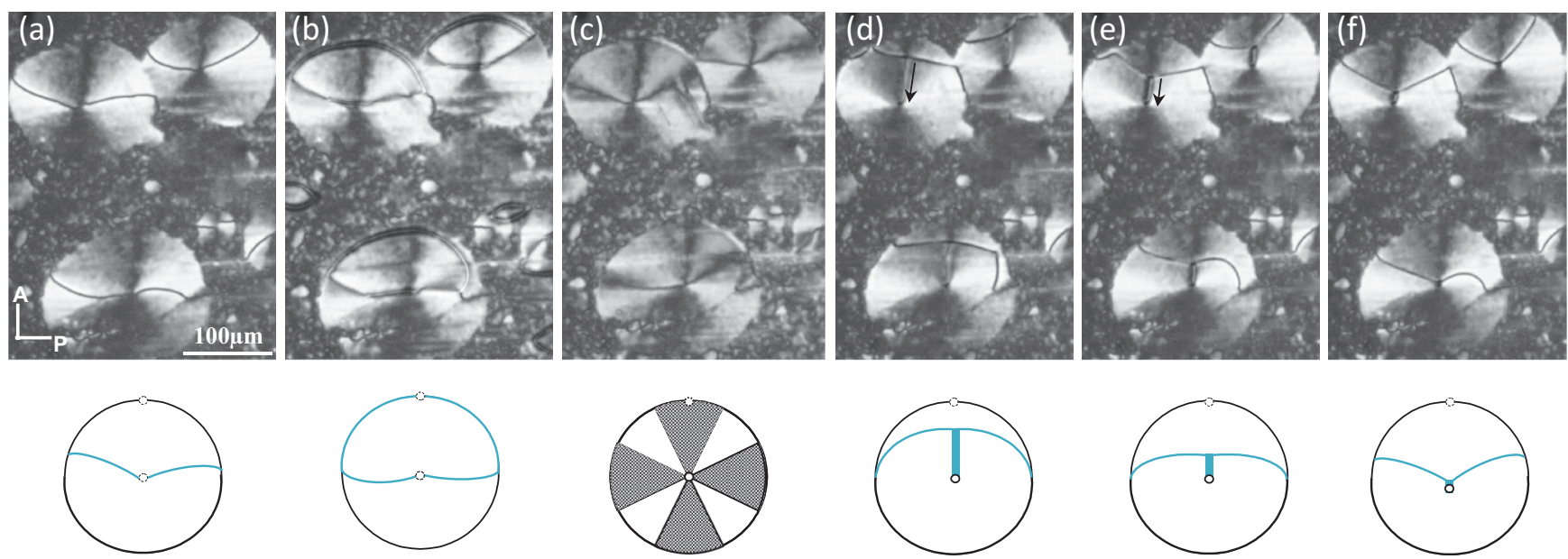

FIG. 9. (Color online) Optical micrograph of a hybrid cell with planar far-field alignment filled with E7. The original single twist-escaped disclinations have spontaneously relaxed into singular bulk disclinations (a). (b, c) A $6 \mathrm{~V}$ ac electric field leads to the transition to a homeotropic configuration through the formation of transient disclination lines and loops. When the field is abruptly switched off, metastable twist-escaped disclinations form and relax as shown after $4.0 \mathrm{~s}$ (d), $5.5 \mathrm{~s}$ (e), and $7.1 \mathrm{~s}$ (f). The LB film has been transferred on APTES-functionalized ITO glass. The cell gap is $5 \mu \mathrm{m}$. A sketch indicating the location of the two boojums and the visible disclinations inside the top-left domain is included below each micrograph. See Video9 in the supplemental material [34].

boundary conditions (Fig. 10). Twist is maximum in the vicinity of the disclination, where the director is twisted roughly half a turn across the gap, and is zero across the domain diameter [Fig. 10(a)]. By splitting the nonsingular integer twist disclination into two singular semi-integer disclinations, twist distortion is completely relaxed in the vicinity of the original disclination [Fig. 10(a)]. Growing of the region surrounded by the singular disclinations leads to a progressive decrease of the overall twist distortion at the expense of an increase in the length of the disclinations, i.e., an increase of the core energy. The minimum twist distortion would be obtained when the singular disclination spans the domain diameter [Fig. 10(c)]. However, this is not always realized

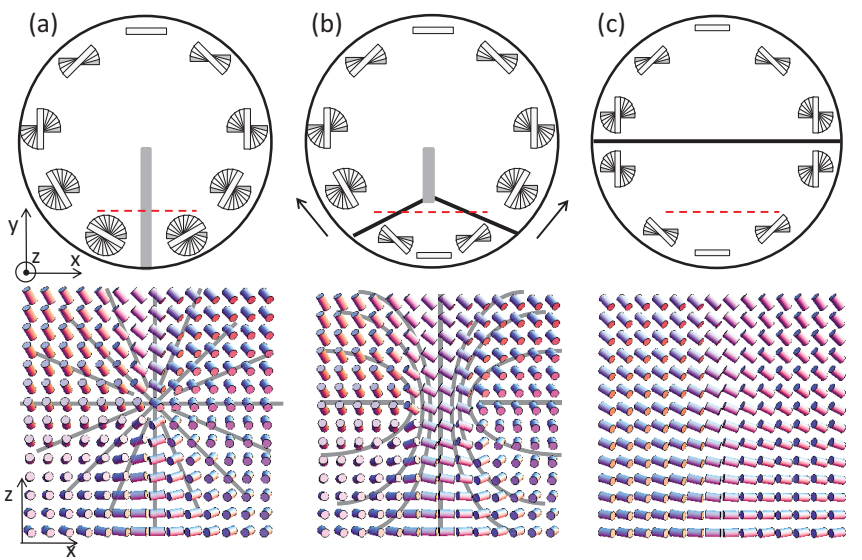

FIG. 10. (Color online) Scheme illustrating the splitting of the twist-escaped integer disclination (a) into two semi-integer singular bulk disclinations (b) leading to a significant reduction in the overall twist distortion across the thickness of the nematic medium (c). The local twist across the cell gap is illustrated in each panel. An estimation of the nematic director in an $X Z$ section across the disclinations (dashed red segment) is shown below each map of twist distributions. experimentally (Fig. 6), and the steady-state configuration features singular disclinations of different shapes in each domain, presumably because of pinning effects on the underlying surfactant monolayer. We have attempted a semiquantitative analysis to compare the elastic energies between the two configurations, but the result is not conclusive. Using a single-elastic constant approximation, we can estimate the elastic energy cost of the semi-integer disclination as

$$
F_{1 / 2} \simeq \frac{1}{4} \pi K L_{1 / 2} \ln \frac{h}{2 r_{c}}+F_{\text {core }},
$$

and that of the nonsingular integer disclination as

$$
F_{1} \simeq 3 \pi K L_{1}
$$

Here, $K$ is the average Frank elastic constant, $L_{1 / 2}$ and $L_{1}$ are the lengths of the disclinations, $h$ is the cell thickness, $r_{c}$ is the core size of the singular disclination, and $F_{\text {core }}$ is its associated elastic energy. Typical core sizes are in the nm range. Since our cell thickness is in the micrometer range, we take the ratio between the two length scales to be of the order $10^{3}$. If we neglect the contribution of the core energy, we estimate

$$
\frac{F_{1 / 2}}{F_{1}} \simeq 0.6 \frac{L_{1 / 2}}{L_{1}} .
$$

Taking into account that the semi-integer disclination is roughly twice the length of the parent nonsingular one, we conclude that the two energies have a similar order of magnitude. A more accurate analysis would thus be required to account for the observed result that semi-integer disclinations are more stable. By taking a typical value for the elastic constant of the mesogens employed here, $K \simeq 10 \mathrm{pN}$, and typical disclination lengths for the cellular domains reported in this work, we get an estimate for the total distortion energy of order $10^{-15} \mathrm{~J}$, showing that thermal fluctuations play no role in the reported processes. 


\section{SUMMARY AND CONCLUSIONS}

We have reported experiments in which we employ an electric field to modulate submillimeter cellular patterns in nematic liquid crystal cells built with hybrid boundary conditions. Interaction during cell filling between the nematic flow field and the ordered surfactant monolayer leads to a defect healing scenario that includes persistent boojum defects and confined bulk disclinations. Only when the mesogen is transited to the isotropic phase and back into the nematic is the memory of the original boojum defects erased. By combining different far-field alignment conditions, orientation of the electric field, and dielectric anisotropy of the mesogen, we can reversibly tune the mesoscale order. In particular, we have focused on the configuration featuring a single twist-escaped disclination that we show to be metastable with respect to relaxation of the twist distortion into singular bulk twist disclinations. A combination of polarizing optical microscopy and fluorescence confocal polarizing microscopy has allowed us to elucidate the transition between the two configurations and to put into evidence the relevance of the boojum defects generated during flow-filling. These two configurations can be interconverted through cycling to electric-field-induced homeotropic alignment, depending on the steepness of the subsequent field quench.

The current work combines the robustness of liquid crystal patterning by contact with monomolecular films of surfactants featuring long-range tilt-orientational order at equilibrium with the ability to reversibly select distinguishable mesoscopic configurations by means of an electric field. Given the rich polymorphism of ordered phases in surfactant monolayers, and the possibility of combining with mesogens with reduced symmetries, our result paves the way for additional strategies to build tunable materials in which self-assembly is mediated by topological defects.

\section{ACKNOWLEDGMENTS}

We acknowledge funding from MICINN (Project FIS201021924-C02-01) and DURSI (Project 2009 SGR 1055).
[1] M. Kléman and O. D. Lavrentovich, Soft Matter Physics: An Introduction, Partially Ordered Systems (Springer, New York, 2003).

[2] P. M. Chaikin and T. C. Lubensky, Principles of Condensed Matter Physics (Cambridge University Press, Cambridge, 1995).

[3] G. Alexander, B. Chen, E. Matsumoto, and R. Kamien, Rev. Mod. Phys. 84, 497 (2012).

[4] P. Poulin, H. Stark, T. C. Lubensky, and D. A. Weitz, Science 275, 1770 (1997).

[5] T. Lopez-Leon, V. Koning, K. B. S. Devaiah, V. Vitelli, and A. Fernandez-Nieves, Nat. Phys. 7, 391 (2011).

[6] T. Lopez-Leon, A. Fernandez-Nieves, M. Nobili, and C. Blanc, Phys. Rev. Lett. 106, 247802 (2011).

[7] J. C. Loudet, P. Barois, and P. Poulin, Nature (London) 407, 611 (2000).

[8] I. Muševič, M. Škarabot, U. Tkalec, M. Ravnik, and S. Žumer, Science 313, 954 (2006).

[9] C. P. Lapointe, T. G. Mason, and I. Smalyukh, Science 326, 1083 (2009).

[10] Q. Liu, B. Senyuk, M. Tasinkevych, and I. Smalyukh, Proc. Natl. Acad. Sci. (USA) 110, 9231 (2013).

[11] B. Senyuk, Q. Liu, S. He, R. D. Kamien, R. B. Kusner, T. C. Lubensky, and I. Smalyukh, Nature (London) 493, 200 (2013).

[12] U. Tkalec and I. Muševič, Soft Matter 9, 8140 (2013).

[13] I. Smalyukh, Y. Lansac, N. A. Clark, and R. P. Trivedi, Nat. Mater. 9, 139 (2010).

[14] A. Honglawan, D. A. Beller, M. Cavallaro, Jr., R. D. Kamien, K. J. Stebe, and S. Yang, PNAS 110, 34 (2013).

[15] T. A. Wood, J. S. Lintuvuori, A. B. Schofield, D. Marenduzzo, and W. C. Poon, Science 334, 79 (2011).

[16] A. Nych, U. Ognysta, M. Skarabot, M. Ravnik, S. Zumer, and I. Musevic, Nat. Commun. 4, 1489 (2013).

[17] D. A. Beller, M. A. Gharbi, A. Honglawan, K. J. Stebe, S. Yang, and R. D. Kamien, Phys. Rev. X 3, 041026 (2013).

[18] Y. Choi, T. Atherton, S. Ferjani, R. G. Petschek, and C. Rosenblatt, Phys. Rev. E 80, 060701 (2009).
[19] T. Ohzono and J. Fukuda, Nat. Commun. 3, 701 (2012).

[20] J. H. Kim, M. Yoneya, and H. Yokoyama, Nature (London) 420, 159 (2002).

[21] J.-i. Niitsuma, M. Yoneya, and H. Yokoyama, J. Appl. Phys. 111, 103507 (2012).

[22] A. Martinez, H. C. Mireles, and I. Smalyukh, Proc. Natl. Acad. Sci. (USA) 108, 20891 (2011).

[23] M. E. McConney, A. Martinez, V. P. Tondiglia, K. M. Lee, D. Langley, I. I. Smalyukh, and T. J. White, Adv. Mat. 25, 5880 (2013).

[24] M. Nespoulous, C. Blanc, and M. Nobili, Phys. Rev. Lett. 104, 097801 (2010).

[25] J. Fang, U. Gehlert, R. Shashidar, and C. M. Knobler, Langmuir 15, 297 (1999).

[26] A. D. Price and D. K. Schwartz, J. Phys. Chem. B 111, 1007 (2007).

[27] V. S. U. Fazio, L. Komitov, and S. T. Lagerwall, Liq. Cryst. 24, 427 (1998).

[28] W. Iglesias, T. J. Smith, P. B. Basnet, S. R. Stefanovic, C. Tschierske, D. J. Lacks, A. Jakli, and E. K. Mann, Soft Matter 7, 9043 (2011).

[29] N. Petit-Garrido, R. P. Trivedi, J. Ignés-Mullol, J. Claret, C. Lapointe, F. Sagués, and I. I. Smalyukh, Phys. Rev. Lett. 107, 177801 (2011).

[30] M. C. Petty, Langmuir-Blodgett Films: An Introduction (Cambridge University Press, Cambridge, 1996).

[31] J. Ignés-Mullol, J. Claret, R. Albalat, J. Crusats, R. Reigada, M. T. M. Romero, and F. Sagués, Langmuir 21, 2948 (2005).

[32] C. A. Schneider, W. S. Rasband, and K. W. Eliceiri, Nat. Methods 9, 671 (2012).

[33] P. Oswald and P. Pieranski, Nematic and Cholesteric Liquid Crystals: Concepts and Physical Properties Illustrated by Experiments (Taylor and Francis, Boca Raton, FL, 2005).

[34] See Supplemental Material at http://link.aps.org/supplemental/ 10.1103/PhysRevE.89.052510 for video files corresponding to the phenomena described in Figs. 4, 5, and 9. 